\title{
Episodic knowledge and implicit learning
}

\author{
ANDREW NEAL \\ University of Queensland, Brisbane, Queensland, Australia \\ and \\ BERYL HESKETH \\ Macquarie University, North Ryde, New South Wales, Australia
}

\begin{abstract}
Despite nearly 20 years of controversy, researchers are still divided over the contributions of abstract and episodic knowledge to performance on implicit learning tasks, and whether these types of knowledge exert their effects unintentionally or unconsciously. The present paper reviews the evidence taken to support the episodic account of implicit learning. Although substantial evidence suggests that episodic knowledge influences performance on implicit learning tasks, at present there are no techniques for testing whether these influences occur outside of awareness. It is argued that future research should investigate whether the retrieval of episodic knowledge in implicit learning tasks is insensitive to intentional control, rather than focus on the issue of awareness. Preliminary evidence suggesting that episodic knowledge can exert a nonintentional influence on task performance is reviewed, followed by a discussion of the status of consciousness as an explanatory construct in psychology.
\end{abstract}

Proponents of implicit learning claim that implicit learning reflects the acquisition of abstract knowledge regarding the statistical regularities within a learning environment, that it occurs outside of awareness, and that it is insensitive to intentional control (Reber, 1989). Such claims are extremely controversial. Critics of the implicit learning literature claim that subjects do not use abstract knowledge in implicit learning tasks (Vokey \& Brooks, 1992) and that learning does not occur outside of awareness (Perruchet \& Pacteau, 1990). The three major reviews of implicit learning published during 1994 reflect the extent of disagreement over the status of the field. On the one hand, Seager (1994) and Berry (1994) both supported the traditional view of implicit learning, although Berry also acknowledged that this view may need to be modified to accommodate the major criticisms of the field. On the other hand, Shanks and St. John (1994) were extremely critical of the traditional view, arguing that there is virtually no evidence to support the existence of implicit learning at all.

Rather than provide another comprehensive review of the implicit learning literature, in the present paper we examine the evidence for one particular account of implicit learning, which has been relatively neglected in most reviews to date. The model examined in the current paper is termed the episodic account of implicit learning. The episodic account assumes that subjects perform implicit learning tasks by reference to prior learning episodes (epi-

We thank Bruce Whittlesea, Robert Mathews, Pierre Perruchet, and Henry L. Roediger III for helpful comments on a previous version of this manuscript. Correspondence regarding this article should be sent to A. Neal at the Department of Psychology, University of Queensland, Brisbane, QLD 4072, Australia (e-mail: andrew@psy.uq.edu.au). sodic knowledge), rather than abstract representations. Although it has long been known that episodic knowledge may influence performance on implicit learning tasks (Brooks, 1978), it has commonly been assumed that these episodic influences must be explicit (e.g., McAndrews \& Moscovitch, 1985; Perruchet \& Pacteau, 1990; Shanks \& St. John, 1994). More recently, however, several researchers have claimed that episodic knowledge can exert an implicit influence on task performance (Higham \& Vokey, 1994; Neal, Hesketh, \& Andrews, 1995; Whittlesea \& Dorken, 1993a). Here we examine whether episodic knowledge can be used to perform implicit learning tasks, and whether these uses of episodic knowledge occur outside of awareness or intentional control. Our primary focus will be on evidence derived from categorization tasks, such as artificial grammars. We will not review the evidence taken to support the traditional abstractive account of implicit learning (e.g., incidental learning and transfer to stimuli with dissimilar characteristics), because the limitations of this evidence have been extensively reviewed elsewhere (Brooks \& Vokey, 1991; Perruchet, 1994a; Perruchet, Gallego, \& Pacteau, 1992; Vokey \& Brooks, 1994; Whittlesea \& Dorken, 1993a).

In a typical artificial grammar learning experiment, the subjects are asked to memorize a series of letter strings that have been generated by a synthetic grammar. In the test phase, the subjects are given a new set of strings and are asked to discriminate the grammatical items from the nongrammatical items. Subjects typically classify between $60 \%$ and $80 \%$ of the items correctly, but they perform very poorly on self-report measures of declarative knowledge (see Reber, 1989, for a review). The evidence suggesting that subjects use episodic knowledge to perform these types of categorization tasks is reviewed below. 


\section{Evidence for Episodic Representation}

\section{Differences Between Abstract and \\ Episodic Representation}

A representation is said to be abstract if it summarizes information across a series of separate learning episodes, rather than simply recording the details of a single episode. Abstract representations, therefore, do not preserve the details of individual processing episodes. Episodic representations, on the other hand, do preserve the details of specific processing episodes and do not summarize information. The basic assumptions of episodic models are that (1) each processing episode is stored as a separate trace, $(2)$ the memory trace represents the processing operations that were carried out within the particular encoding context, and (3) the retrieval of the trace is dependent on the ability of a cue to reinstate the original encoding context (Morris, Bransford, \& Franks, 1977; Tulving \& Thomson, 1973).

Episodic accounts make several predictions regarding task performance and the nature of knowledge representation, which differ from those made by abstractive accounts (Jacoby \& Brooks, 1984; Whittlesea \& Dorken, 1993a). First, an episodic account suggests that task performance should be sensitive to the details of the original processing episodes, even if those details are nominally irrelevant. In contrast, abstractive accounts tend to emphasize the stability of abstract knowledge across nominally irrelevant contextual factors. Second, episodic accounts emphasize the similarities between abstract and episodic knowledge, since both types of knowledge are thought to be stored in a common memory system and are thought to be governed by the same principles. Abstractive accounts, by contrast, treat episodic and abstract knowledge as qualitatively different and assume that they are stored in separate memory systems (e.g., Knowlton, Ramus, \& Squire, 1992). Third, episodic accounts suggest that there is no mechanism that automatically abstracts structural information. Abstract information can be stored in an episodic system only if the learner carries out abstraction at encoding intentionally. Many abstractive accounts assume that abstraction occurs automatically whenever one interacts with a structured stimulus environment (e.g., Mathews \& Roussel, 1993; Posner \& Keele, 1968; Reber, 1989; Servan-Schreiber \& Anderson, 1990).

Evidence to support the episodic view comes from five sources of findings, showing that: (1) analogic strategies appear to be more common than proponents of the abstractive account would expect, (2) episodic systems can perform abstraction, (3) episodic models can account for the relationships between categorization and recognition, (4) a wide range of tasks that were previously thought to rely on abstract knowledge are sensitive to encoding specificity effects, and (5) abstraction is under the control of task demands and is not an automatic consequence of attending to a structured stimulus environment. The evidence from each of these five sources is reviewed below.

\section{Evidence for Episodic Representation}

Analogic strategies are common. Brooks (1978) produced the first evidence in support of the episodic account when he demonstrated that subjects can discriminate grammatical from nongrammatical test strings on the basis of the similarity between the test string and a previously encountered training string. Most proponents of implicit learning have subsequently acknowledged that subjects can use an analogic strategy to perform the string discrimination task. However, these proponents have argued that the use of analogic strategies is confined to unusual situations, such as paired associate learning, which emphasize the memorization of the individuating features of each item (Reber \& Allen, 1978).

Vokey and Brooks (1992) demonstrated that the use of analogy is not restricted to conditions that place an unusually strong emphasis on memorization. Vokey and Brooks (1992) manipulated similarity to specific training items and grammatical membership orthogonally within their stimulus set, and found that similarity influenced performance across a variety of training conditions, including standard implicit learning conditions. The existence of similarity effects under standard implicit learning conditions discredits Reber and Allen's (1978) argument that analogic strategies are confined to unusual training tasks.

Episodic systems can perform abstraction. Vokey and Brooks (1992) also found that grammatical membership influenced performance, and that the effects of similarity and grammatical membership were independent of each other. Proponents of implicit learning have argued that the effect of grammatical membership supports their claim that implicit learning produces abstract knowledge (e.g., McAndrews \& Moscovitch, 1985; Seager, 1994). However, Vokey and Brooks (1992) argue that the independent effects of similarity and grammaticality may reflect differences in the retrieval of information from a common knowledge base, rather than the use of separate types of knowledge. Episodic models of categorization (Hintzman, 1986; Medin \& Shaffer, 1978; Nosofsky, 1986) assume that categorization decisions are made with reference to a pool of items (or "chorus of instances") in memory. Although the pooling of items represents a form of abstraction, this abstraction is very different from that proposed by traditional abstractive models. The episodic models assume that abstraction occurs at retrieval, rather than at encoding, or during storage.

Hintzman's (1986) MINERVA2 model, for example, assumes that each memory trace is compared with the test item (in parallel) and is given a certain level of activation. The degree to which each trace is activated is a function of its similarity with the test instance. The activation functions are nonlinear, so that the traces that are most similar have disproportionately high levels of activation, and items that are dissimilar exert virtually no effect. The items that have been activated collectively form a pool of items, and the features within this pool form the standard against which the test item is compared. If the degree of match between the pool of activated items and 
the test item exceeds the criterion, the item is classified as grammatical.

These "chorus of instances" models can account for the effects of both similarity and grammaticality. Categorization decisions will be sensitive to similarity if the pool is dominated by a single item. The nonlinear similarity gradients allow a single item to exert a disproportionately large effect within the pool if it is sufficiently distinctive. On the other hand, the categorization decision will be sensitive to grammaticality if the pool consists of a number of items. If a number of traces have been activated, the prototypical features will tend to dominate within the pool, and the decision will reflect sensitivity to those structural features. For this reason, episodic models can account for grammaticality effects without requiring the storage of abstract knowledge. If abstraction occurs at retrieval, there is no need to assume the existence of separate learning systems, or knowledge bases. ${ }^{1}$

Relationships between categorization and recognition. Proponents of abstractive accounts of implicit learning have argued that performance on categorization tasks should be dissociated from performance on recognition tasks, because categorization requires the use of abstract knowledge, and recognition requires the use of episodic knowledge. Perhaps the strongest evidence to support this claim was produced by Knowlton et al. (1992), who found that amnesics performed as well as control subjects when instructed to sort test items according to categorical membership. However, amnesics were worse than controls on tests of recognition memory, as well as when they were instructed to sort the test items on the basis of similarity to prior items. Knowlton et al. argued that single-factor episodic models cannot explain these findings, because they predict that categorization performance should always be associated with recognition performance. Knowlton et al., therefore, concluded that these results demonstrate that abstract knowledge contributes to categorization performance, and that the use of abstract knowledge is independent of the explicit use of prior examples.

Knowlton et al's (1992) conclusion is problematic for several reasons. First, several commentators (e.g., Jacoby, 1991; Reingold \& Merikle, 1988) have pointed out that task dissociations do not represent evidence for the existence of separate learning or memory systems (see below). Second, even if one accepts that the dissociation between categorization and recognition in the amnesic group demonstrates that subjects can classify items without using explicit memory for prior instances, this does not demonstrate that they have used abstract knowledge to classify the items. There is evidence to suggest that episodic knowledge may exert an implicit influence on categorization performance (see below). Finally, singlefactor episodic accounts can actually account for dissociations between categorization and recognition. This final problem is discussed below.

Episodic models predict that the relationship between categorization and recognition will vary according to the nature of the similarity relationships between the stimuli. The context model of categorization (Medin \& Schaffer,
1978; Nosofsky, 1986), for example, assumes that categorization decisions are made by comparing (1) the similarity between the test item and all of the items in its own category with (2) the global similarity between the test item and all items in all categories. Recognition decisions, on the other hand, are simply a function of the global similarity between the test item and all items in all categories. Figure 1 illustrates a situation in which these two decision rules will produce a dissociation between categorization and recognition (Medin, 1986). A series of exemplars from two categories (A and B) are shown on a two-dimensional plane. The similarity between any two items on this plane is assumed to be a decreasing exponential function of the distance between the items. The new item $\left(N_{1}\right)$ should be reliably categorized as a member of $\mathrm{B}$, because it is more similar to the items in category B than to items in category A. However, $N_{1}$ is also likely to be falsely recognized as old, because it is similar to a number of old items. Categorization, therefore, will be dissociated from recognition.

Evidence supporting this analysis was provided by Medin, Dewey, and Murphy (1983), who demonstrated that increasing the distinctiveness of individual training items, by associating exemplar level information with each item, reduced categorization accuracy. The context model can explain this apparently counterintuitive finding, because it assumes that increasing the distinctiveness of the training items will decrease the similarity between the training and test items. Since the similarity gradients are nonlinear, the decrease in similarity will be stronger

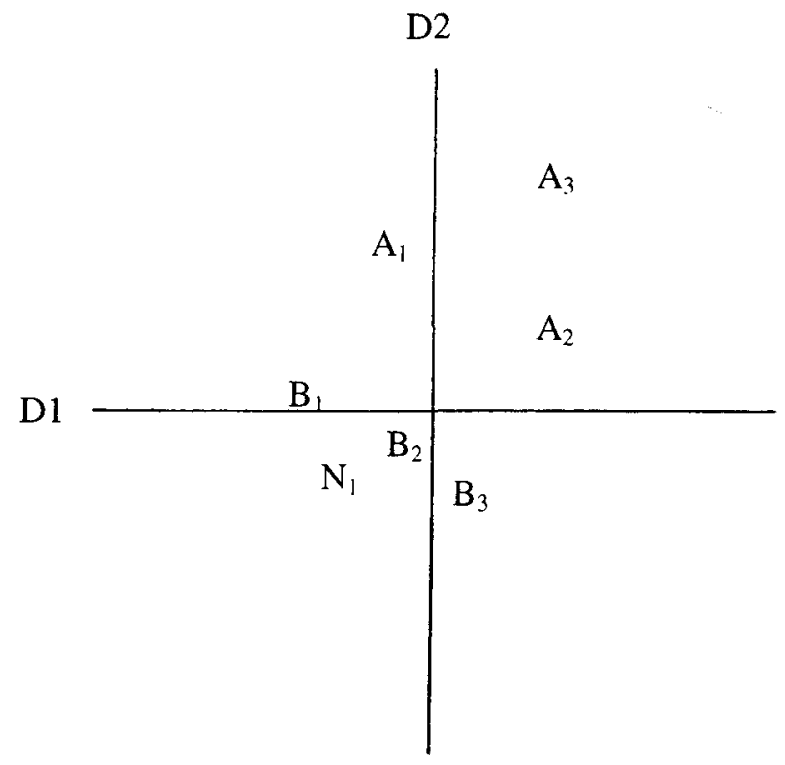

Figure 1. Spatial representation of a set of training exemplars $\left(A_{1}, A_{2}, A_{3}, B_{1}, B_{2}, B_{3}\right)$ from two categories ( $A$ and $B$ ), and a previously unseen test item $\left(N_{1}\right)$. From "Comment on 'Memory Storage and Retrieval Processes in Category Learning,"' by D. $L$. Medin, 1986, Journal of Experimental Psychology: General, 115, p. 376. Copyright 1986 by the American Psychological Association. Adapted with permission. 
for same-category items than for opposite-category items, and categorization performance will be impaired.

Medin et al.'s (1983) data illustrate how the context model can explain the dissociation between categorization and recognition reported by Knowlton et al. (1992). If amnesia reduces the richness or distinctiveness of the items in memory, then it will increase the relative similarity between new test items and old training items. This increase in similarity will impair recognition, because it will cause the subjects to make false alarms to previously unseen items. On the other hand, this increase in similarity might actually increase categorization accuracy, because the increase in similarity should be greater for items in the same category than for items in the opposite category. The context model, therefore, can actually predict a stronger dissociation between categorization and recognition than that found by Knowlton et al. These findings demonstrate that dissociations between categorization and recognition are not inconsistent with singlefactor episodic models.

Abstractive models, on the other hand, have difficulty accounting for the effects of distinctiveness on the relationship between categorization and recognition. Abstractive models suggest that increasing the distinctiveness of training items should produce greater reliance on episodic knowledge and less reliance on abstract knowledge (Reber \& Allen, 1978). If this were the case, then mnemonic training conditions, which increase item individuation, should increase the effects of similarity and reduce the effect of grammaticality. However, Vokey and Brooks (1992, Experiments 2 and 3) found that mnemonic training conditions decreased the effect of similarity, not grammaticality. As argued previously, episodic models predict that increasing the distinctiveness of the individual items will decrease the effect of similarity, because it emphasizes relatively minor differences between the stimuli.

Abstractive models also have difficulty accounting for the effect of test phase instructions on the use of similarity and grammaticality. Vokey and Brooks (1992) argued that if subjects use abstract knowledge to perform categorization tasks and use episodic knowledge to perform recognition tasks, then (1) similarity should exert a stronger effect on recognition than grammaticality should, and (2) grammaticality should exert a stronger effect on categorization than similarity should. Furthermore, instructing the subjects to sort the strings on the basis of grammaticality, rather than similarity, would be expected to increase the effect of grammaticality (see Knowlton et al., 1992, for a similar argument). However, Vokey and Brooks (1992, Experiment 1) found that (1) grammaticality exerted a stronger effect on recognition than did similarity, (2) grammaticality and similarity exerted the same influence on categorization performance, and (3) instructing subjects to sort the stimuli on the basis of grammaticality did not increase the effect of grammaticality. These findings demonstrate that grammaticality and similarity are not sensitive to the types of manipulations that one would expect if grammaticality and similarity reflected the use of separate knowledge bases. Collectively, these particular findings lend support to a single-knowledge model, rather than a dual-knowledge model.

Encoding specificity effects. The next source of evidence that supports the episodic approach stems from demonstrations of encoding specificity effects. Encoding specificity refers to the sensitivity of task performance to the match between the demands of encoding and retrieval tasks (Tulving \& Thomson, 1973). Encoding specificity effects, which are diagnostic of episodic knowledge representation, have been found across a wide variety of tasks that were previously thought to rely on abstraction. Although encoding specificity effects are not necessarily incompatible with abstract knowledge representation, abstractive models tend to emphasize the stability of knowledge in the face of contextual variation. If tasks that are commonly assumed to rely on abstract knowledge are sensitive to encoding specificity effects, this suggests that these "abstractive" tasks are governed by the same principles as are traditional episodic tasks such as recall and recognition. Such findings question the need to assume that abstract and episodic knowledge are qualitatively different.

Perceptual identification is commonly assumed to reflect the use of abstract knowledge structures, such as logogens (Morton, 1969), yet Jacoby, Baker, and Brooks (1989) demonstrated that perceptual identification is sensitive to the same encoding and retrieval effects as is recall, which is an episodic task. Jacoby et al. (1989) found that exposure to a picture can facilitate performance on subsequent perceptual identification and recall tasks, and that the degree of facilitation was a function of the degree of match between the processing demands of the encoding and retrieval tasks. Furthermore, the effects of prior experience generalized to new items of the same category, and the degree of generalization was influenced by the demands of the original processing task. The parallel effects of context on the perceptual identification and recall tasks is not easily accounted for by an abstractive model (see also Jacoby, 1983).

Some evidence of encoding specificity effects has also been found with the use of artificial grammar learning tasks. Vokey and Brooks (1992, Experiment 4) found that the effect of similarity was sensitive to the match between encoding and retrieval conditions. Context was manipulated by pairing a phrase with each letter string. Similarity influenced performance only when the retrieval context matched the encoding context. Unfortunately, however, grammaticality was not sensitive to the match between encoding and retrieval conditions. One might expect that if grammaticality and similarity both reflected the operation of a common episodic system, both should be sensitive to encoding specificity effects.

Stronger evidence for encoding specificity effects has been reported by Whittlesea and Dorken (1993a, Experiments 1 and 2), who found that the encoding task itself can influence performance, independently of the features of the exemplars. The training items were generated by two separate grammars. Subjects were asked to pronounce 
the items from one grammar and spell the items from the other. In the test phase, subjects had to spell or pronounce the items and to indicate the grammar to which they belonged. Classification performance was better when the demands of the test task matched those of the encoding task, even if the item itself was equally similar to items from both categories. The sensitivity of categorization decisions to encoding specificity effects questions the traditional emphasis on stable abstractions as explanations of categorization.

Finally, there is also evidence to suggest that rule use may be sensitive to the match between encoding and retrieval contexts. Rothkopf and Dashen (1995) demonstrated that subjects could use a rule to solve problems more quickly if the problems were generated with the same sets of features as those of the original training items than if the problems were generated with a broader set of features. Similarly, Neal et al. (1995) found that estimates for rule use in a categorization task were higher for previously seen items than for new items. In both studies the rules exerted a stronger influence on the old items than on the new, despite the fact that these rules were equally applicable to both types of items. These findings suggest that rules may not form a stable body of decontextualized knowledge, as is suggested by abstractive accounts, but rather that they become embedded within the context in which they are practiced. The apparent contextual sensitivity of rule knowledge further questions the need to draw qualitative distinctions between abstract and episodic knowledge.

In summary, these findings suggest that episodic knowledge exerts a far stronger influence on a wide range of tasks than was previously thought, and they add plausibility to the claim that implicit learning may reflect the acquisition of episodic knowledge. However, as stated previously, encoding specificity effects are not necessarily incompatible with abstractive accounts. Mathews and Roussel (1993) point out that modern (fragmentary) abstractive models (e.g., Servan-Schreiber \& Anderson, 1990 ) assume that knowledge is abstracted from representations stored in episodic memory and therefore may be sensitive to encoding specificity effects. However, if abstraction does occur during storage, the summary representation should lose the contextual detail associated with the individual learning episodes (Whittlesea \& Dorken, 1993b). If this were the case, contextual cues would no longer be effective at retrieval, and categorization should not be sensitive to encoding specificity effects.

The final source of evidence that supports episodic models of implicit learning stems from findings which demonstrate that abstraction is under the control of task demands and is not an automatic consequence of attending to a structured stimulus environment.

Abstraction is under the control of task demands. Since episodic models assume that the content of a memory trace is a function of the processing operations that were carried out at encoding, they predict that abstraction should be under the control of task demands (Whittlesea \& Dorken, 1993a). According to this view, abstrac- tion should occur only when demanded by the task at hand. Abstractive models of implicit learning, however, assume that abstraction is an automatic consequence of attending to any structured stimulus display (e.g., Reber, 1989). Abstractive models of implicit learning, therefore, predict that abstraction should occur regardless of whether it is demanded by the task at hand.

Whittlesea and Dorken (1993a, Experiment 5) tested the hypothesis that abstraction is sensitive to task demands in three incidental training tasks: one in which the stimuli were merely distractors in a number learning experiment, one that required the subjects to encode structural relationships within the stimuli (but was still an incidental task), and one that required the subjects to memorize the stimuli. Each incidental task produced different patterns of performance on same-letter and different-letter transfer tests, suggesting that knowledge representation was under the control of the encoding task. The task in which the stimuli were presented as distractors produced transfer on the same-letter set but not on the differentletter set, suggesting that the subjects only acquired knowledge relating to the surface characteristics of the stimuli. The task that required encoding of structural relationships produced equally good transfer on both tests, suggesting that the subjects acquired deep structural knowledge. The task that required memorization produced transfer on both tests, but performance was better on the same-letter set than on the different-letter set, suggesting that the subjects had only acquired shallow structural knowledge. These findings demonstrate that abstraction is sensitive to the demands imposed by the encoding task, and they discredit the assumption that abstraction occurs automatically whenever the learner attends to a stimulus display.

Similar findings were reported by Whittlesea (1987), who found that test items were classified on the basis of their similarity to the category prototype (an effect termed the "goodness of exemplar effect") when the encoding task required subjects to analyze the stimuli into subunits. However, the test items were classified on the basis of their similarity to specific old items (an effect termed the "closeness to old effect") when the stimuli were processed as integral units. These findings suggest that abstraction occurs only when the encoding task requires it. If the encoding task is analytic, abstract information will be stored, not because abstraction occurs automatically, but because the information in memory represents a record of the processing episode as it was experienced.

Wattenmaker (1993) provides further evidence that sensitivity to the structural properties of a category is most likely a by-product of the processing demands imposed by encoding and retrieval tasks, rather than the operation of an automatic abstraction mechanism. Wattenmaker $(1991,1993)$ found that subjects could acquire sensitivity to correlated features within a category, even when they were unaware of the correlations at encoding. Most abstractive models of categorization have difficulty accounting for the acquisition of correlated features (Medin \& Schwanenflugel, 1981). Episodic models can, however, 
account for the learning of correlated feature sets. Categorization decisions that are based on similarity are likely to be accurate, because the stimuli that preserve the correlation between features are more likely to resemble each other than items that do not preserve the correlation. These data suggest that structural information can be preserved indirectly in the exemplars and does not have to be directly abstracted in order for it to influence subsequent performance. Furthermore, different incidental tasks had different effects on performance, suggesting that the form in which information was represented was a function of the demands of the encoding tasks.

Collectively, the findings reviewed thus far lend relatively strong support to the episodic approach (see also Dienes \& Berry, 1997). Rather than being under the control of the nominal status of a task (requiring item- or category-level responses), performance appears to be under the control of the processing demands of a task. Knowledge representation appears to be critically dependent on the demands of the encoding task, and performance is strongly influenced by the match between encoding and retrieval conditions.

\section{Evidence for Fragmentary Representation}

The fragmentary knowledge account is the other major alternative explanation of implicit learning phenomena. Proponents of the fragmentary knowledge account assume that subjects do not learn the "deep" structure of an artificial grammar. Instead, it is thought that subjects acquire fragmentary and incomplete knowledge regarding permissible groups or sequences of letters (Dulany, Carlson, \& Dewey, 1984; Perruchet, 1994b). This fragmentary knowledge is assumed to consist mostly of knowledge regarding which bigrams are permissible. According to this account, there is no need to assume the existence of any abstractive mechanism, because subjects can classify strings as grammatical if they recognize individual fragments within the items (Perruchet, 1994b).

Evidence to support the fragmentary knowledge account comes from findings demonstrating that (1) classification performance is often associated with fragmentary knowledge, (2) classification performance can be simulated on the basis of fragmentary knowledge, and (3) subjects who are trained on isolated bigrams perform equivalently to subjects who are given training with the full strings.

A number of papers have reported associations between classification performance and fragmentary knowledge. Dulany et al. (1984) measured fragmentary knowledge by asking subjects to underline the part of the item which made it grammatical or not. Dulany et al. found that the validity of the fragmentary rules which subjects reported correlated with the proportion of correct responses that they made. Dienes, Broadbent, and Berry (1991) also found that classification performance closely mirrored performance on a measure of fragmentary knowledge, termed the sequential letter dependencies task. These associations between classification performance and frag- mentary knowledge are commonly taken to suggest that the two measures tap the same knowledge base.

A number of papers have demonstrated that classification performance can be simulated with data from a test of fragmentary knowledge. Perruchet and Pacteau (1990) simulated classification performance using a decision rule which classified any item as grammatical if the subjects recognized all of the bigrams within the string. Perruchet (1994a) simulated classification performance using a decision rule which classified any item as grammatical if it shared its terminal trigram with at least one training item. In both cases, the simulated data matched actual classification performance. Furthermore, Perruchet and Pacteau (1990) demonstrated that preventing subjects from encoding whole strings by segmenting the training items into bigrams did not harm subseqent performance. Collectively, these findings demonstrate that subjects could rely on bigram or trigram knowledge to perform the string discrimination task. The fragmentary knowledge account, therefore, can explain performance without resorting to the concept of automatic abstraction.

Not all fragmentary knowledge models deny the possibility of automatic abstraction, however. Servan-Schreiber and Anderson's (1990) competitive chunking model retains a role for abstraction, since it assumes that fragmentary knowledge representations summarize information across separate learning episodes. According to the competitive chunking model, subjects naturally break letter strings into chunks of two to three letters. As learning proceeds, these separate chunks of letters are joined together into higher level chunks, and the strength of each chunk is modified according to its frequency of use. This strengthening process represents abstraction, because the prototypical chunks become heavily weighted, and the idiosyncratic details of each episode are lost (see also Mathews, 1991). The categorization decisions are based on the familiarity of the letter strings. If a test item preserves a large proportion of the chunks formed during the training phase, it will be highly familiar and will be judged as grammatical. Servan-Schreiber and Anderson (1990) found that (1) subjects were more likely to judge an item as grammatical if it preserved the chunks that were formed during the training phase, and (2) the competitive chunking model provided an accurate simulation of human performance. Unfortunately, there have been no direct comparisons of the alternative fragmentary knowledge models.

\section{Comparing the Episodic and Fragmentary Knowledge Accounts}

The episodic and fragmentary knowledge accounts are often seen as competing explanations of implicit learning phenomena. Perruchet (1994a) argues that both accounts are similar in that they both assume that performance reflects the use of information retrieved from memory, rather than the use of abstract rules, but that the two accounts differ with respect to their unit of analysis (whole strings vs. fragments). Perruchet (1994a) provides two reasons for viewing the fragmentary knowledge hypoth- 
esis as a more parsimonious account of artificial grammar learning than the episodic approach.

First, Perruchet and Pacteau (1990) use Reber and Allen's (1978) argument that subjects use only an analogic strategy when the training conditions favor the memorization of individual strings to claim that an analogic mechanism is not sufficiently powerful to account for grammar learning. However, as argued previously, the combined weight of the findings of Brooks, Vokey, and Whittlesea suggest that analogic strategies may be used across a wide variety of training and test conditions, and not just with conditions that favour memorization.

Second, Perruchet argues that the episodic model is unable to account for the effect of grammaticality in Vokey and Brooks's (1992) data (Perruchet, 1994a; Perruchet \& Pacteau, 1990). Perruchet (1994a) argues that Vokey and Brooks (1992) adopt a dual-knowledge model, in which they account for the effect of similarity by reference to episodic knowledge, but account for the effect of grammaticality by reference to abstract knowledge. The fragmentary knowledge model, on the other hand, can account for the effects of similarity and grammaticality, without having to rely on abstraction. Perruchet (1994a) reanalyzed Vokey and Brooks's (1992) data and demonstrated that subjects who used trigram knowledge to classify the test strings would produce independent effects of similarity and grammaticality, simply because both the similar and the grammatical items had more trigrams in common with the training items than did the dissimilar and nongrammatical items. The apparent ability of the fragmentary knowledge approach to account for findings that the episodic approach cannot handle appears to make the fragmentary knowledge approach a more powerful and parsimonious explanation of artificial grammar learning.

Perruchet (1994a) also argues that the fragmentary knowledge approach can account for the failure of instructional manipulations to modify subjects' reliance on similarity and grammaticality (Vokey \& Brooks, 1992). If subjects do not use specific similarity or abstract knowledge of the structure of the grammar, then naturally instructions to modify their reliance on these two sources of knowledge should not be effective. In sum, Perruchet (1994a) argues that changing the unit of analysis from whole items to fragments adds to the explanatory power of the memory-based approach, because it allows the memory-based approach to explain all existing findings by reference to a single knowledge base.

Contrary to Perruchet's (1994a) argument, however, the episodic model can account for existing findings by reference to only one knowledge base, and therefore there is no need to change the unit of analysis, as Perruchet suggests. The problem with Perruchet's (1994a) analysis of Vokey and Brooks's (1992) data is that he misinterprets Vokey and Brooks's explanation of grammaticality. Vokey and Brooks (1992) argue that similarity and grammaticality do not reflect the use of separate knowledge bases, but rather reflect the similarity of the test items to specific training items, on the one hand, and to the en- tire pool of training items, on the other. According to this explanation, the empirical effects of similarity and grammaticality are orthogonal merely because similarity to specific instances has been manipulated orthogonally to similarity to the entire pool of training items. On the one hand, Perruchet may be right that Vokey and Brooks have overinterpreted the independence of grammaticality and similarity, in that independence is merely a trivial reflection of the experimental design (in which similarity to specific instances was orthogonal to similarity to the whole pool of items). More importantly, however, both the episodic and the fragmentary knowledge approaches can account for the effects of similarity and grammaticality by reference to a single knowledge base, and a single retrieval process. Changing the unit of analysis from the whole string to smaller units such as trigrams, therefore, does not add to the explanatory power of the episodic model as Perruchet claims.

Furthermore, Perruchet's (1994a) dismissal of the finding that similarity and grammaticality are not under good instructional control is misleading. The original aim of examining the sensitivity of similarity and grammaticality to instructional control was to test the predictions of a dual-knowledge model. As Perruchet (1994a) notes, the failure of the instructional manipulations to modify reliance on similarity and grammaticality discredits the assumption that similarity and grammaticality reflect the operation of qualitatively different types of knowledge and favors a single episodic knowledge account-the very point that Vokey and Brooks (1992) were making.

The emphasis that Perruchet (1994a, 1994b) places on attempting to resolve the question of whether knowledge is represented as small fragments or as whole exemplars may actually limit the generality of the memory-based approach rather than enhance it. An episodic account assumes that the form in which knowledge is represented is a by-product of the original encoding operations, so that a learner can have either fragmentary or unitary representations, depending on the stimulus set and encoding task. The fragmentary knowledge position, therefore, can be explained within the more general framework of the episodic account (Whittlesea \& Dorken, 1993a). The differential effects of mnemonic training conditions on recognition and categorization performance (Vokey \& Brooks, 1992) provides an example of how the emphasis on fragmentary knowledge limits the generality of memory-based explanations. Mnemonic training improved recognition memory for training items, but impaired categorization. The fragmentary knowledge account has difficulty explaining this result, because it assumes that categorization decisions are based on the recognition of fragments within strings (e.g., Perruchet \& Pacteau, 1990). A full episodic account can explain this result, because it provides a principled analysis of the categorization-recognition relationship and the effect of contextual factors on that relationship. The episodic account assumes that categorization and recognition decisions access the same representations, but that the decision processes differ (Nosofsky, 1988). Increasing item 
individuation in mnemonic conditions makes that item more distinctive, and hence improves recognition, but it renders the item less similar to other items, and hence impairs categorization. The fragmentary knowledge account has difficulty with these types of findings, because it does not draw upon the more general principles of episodic memory. Rather than attempt to resolve the issue of whether episodic knowledge is represented as fragments or as whole strings, future research should focus on testing the competing predictions of models that include the concept of automatic abstraction (Mathews, 1991; Servan-Schreiber \& Anderson, 1990) and those that exclude it (Perruchet, 1994b; Whittlesea \& Dorken, 1993a).

Despite the ongoing disagreement regarding the necessity of automatic abstraction, most recent approaches to implicit learning accept that episodic knowledge does exert a strong influence on task performance. Unfortunately, however, very few studies have directly tested whether these influences of episodic knowledge are implicit or explicit. Traditionally, researchers have tried to infer the conscious status of task knowledge by using the logic of task association or dissociation. Unfortunately, there are serious problems with the use of this technique. These problems go beyond the issues raised by past critics of the implicit learning literature. The following section reviews the problems associated with attempting to infer the conscious status of task knowledge.

\section{Problems With Inferring the Conscious Status of Task Knowledge}

Virtually all of the attempts to infer the conscious status of task knowledge in the implicit learning literature have relied on the logic of task association and task dissociation. Proponents of implicit learning have used dissociations between performance and self-report measures of declarative knowledge to demonstrate that task knowledge must be unconscious (e.g., Berry \& Broadbent, 1984; Lewicki, Hill, \& Bizot, 1988; Reber \& Lewis, 1977). Critics of implicit learning have used associations between performance and recognition measures to demonstrate that task knowledge must be conscious (e.g., Perruchet \& Amorim, 1992; Perruchet \& Pacteau, 1990).

Unfortunately, there are serious problems with using the logic of association and dissociation to draw inferences regarding the conscious status of task knowledge. The logic of association and dissociation rests on two assumptions, which have been termed exhaustiveness and exclusiveness (Reingold \& Merikle, 1988). A measure of explicit or implicit knowledge is said to be exhaustive if it captures all of the relevant knowledge of the subject. On the other hand, a measure of explicit or implicit knowledge is said to be exclusive if it is not contaminated by other sources of knowledge. There is strong reason to believe that almost any set of experimental tasks will violate these two assumptions.

Critics of implicit learning have long drawn attention to problems with the exhaustiveness assumption (Brody,
1989; Dulany et al., 1984; Perruchet \& Pacteau, 1990; Shanks \& St. John, 1994). If the measure of explicit knowledge is not exhaustive, it will always be possible to claim that any observed dissociation is misleading, because some unmeasured form of explicit knowledge may be responsible for the learning. Such a claim is not falsifiable, unless one has an exhaustive measure of awareness. Defenders of implicit learning (e.g., Reber, Allen, \& Regen, 1985) have replied by pointing to problems with the exclusiveness assumption. If the measure of explicit knowledge is not exclusive (i.e., if the test is contaminated by implicit knowledge), it will always be possible to claim that any observed association is misleading, because implicit knowledge may be responsible for the association. Such a claim is not falsifiable, unless one has an exclusive measure of awareness.

It is extremely difficult to try to justify the claim that one has satisfied the exhaustiveness criterion. One major problem is that there are no commonly accepted criteria for what constitutes an acceptable measure of awareness (Reingold \& Merikle, 1988). Different commentators have developed their own criteria, and it is difficult to compare the adequacy of these different criteria. For example, although recognition measures reveal knowledge that recall measures fail to detect, it is difficult to decide which measure is superior (Erdelyi, 1986). Holender (1986) argues that the recognition measure is superior because it is the more sensitive of the two. However, sensitivity is not an appropriate criterion. Any attempt to maximize sensitivity runs the risk of losing exclusivity. For example, although recognition measures are more sensitive than recall measures, they are probably contaminated by familiarity. Merikle and Cheesman (1986), on the other hand, argue that subjective measures of awareness are more appropriate than objective measures, because they more closely capture the phenomenological difference between conscious and unconscious experience (see Dienes \& Berry, 1997). Because the different measures produce different results, and there is no objective criterion for choosing a measure in the first place, this type of experimental procedure is empirically indeterminate. One can argue that the results of any study are inconclusive by adopting different criteria for what constitutes an adequate measure of awareness.

It is also extremely difficult to try to justify the claim that one has satisfied the exclusiveness criterion. Some of these difficulties are illustrated by Holender's (1986) attempt to justify the claim that forced choice tests (such as recognition) are exclusive measures of awareness. Holender makes two assumptions to justify his claim. The two assumptions are (1) that voluntary responses require intention, and (2) that intention is a central feature of consciousness. Holender argues that any task is a measure of awareness if it directly asks for a voluntary discriminative response. Since a voluntary response is assumed to require intention, and an intentional response is assumed to be conscious, any voluntary discriminative response must be conscious. However, this argument holds true only if one also assumes that voluntary re- 
sponses exclusively require intention - that is, a voluntary response can never occur in the absence of intention. The argument is, therefore, circular: One needs to make the exclusiveness assumption in order to justify the assumption that voluntary responses require intention, when the assumption that voluntary responses require intention is the basis for justifying the exclusiveness assumption.

One of the major reasons why the exclusivity assumption is unwarranted is that there is substantial evidence to suggest that direct tests, such as recognition, represent a mixture of both explicit and implicit processes (Jacoby, 1991; Merikle \& Reingold, 1991). Dual-process theories of recognition (Jacoby, 1991; Jacoby \& Dallas, 1981; Mandler, 1980) assume that recognition judgments reflect the contribution of both familiarity and recollection. Familiarity is assumed to be an unconscious basis for recognition judgments, which is insensitive to intentional control and dual-task interference. Recollection, on the other hand, is assumed to be a conscious basis for recognition judgments, which is sensitive to intentional control and dual task interference. Dual-process theory demonstrates that it is unreasonable to make an a priori assumption that recognition tests must exclusively measure conscious knowledge.

Jacoby (1991) developed the process dissociation procedure to test dual-process theories of memory. Process dissociation allows one to infer the contribution of intentional and nonintentional processes to task performance, without resting on the assumptions of exclusivity or exhaustivity. The logic of the technique is that the contribution of intentional and nonintentional processes can be inferred by comparing performance in a situation where the processes act together to a situation where the processes act in opposition. Jacoby, Toth, and Yonelinas (1993), for example, gave subjects a stem completion task. In the inclusion condition, the subjects were told to complete the stems with items from a previous study list. In the exclusion condition, however, the subjects were told to complete the stems with items that were not on the study list. The difference between performance in the inclusion and exclusion conditions provides a measure of intentional uses of memory. If subjects can consciously recollect the study items, they should include those items in the inclusion condition and exclude the items in the exclusion condition. Nonintentional forms of retrieval, on the other hand, can be indexed by the proportion of study items generated in the exclusion condition (false alarms). Such false alarms cannot be caused by recollection of the instances, because conscious recollection would allow the subject to exclude the item.

Jacoby and his colleagues have demonstrated that (1) secondary tasks impair recollection, but not familiarity, (2) increasing depth of processing benefits recollection more than familiarity, (3) disrupting the perceptual similarity between training and test disrupts familiarity more than recollection, and (4) age-related memory deficits are more severe for recollection than for familiarity (Debner \& Jacoby, 1994; Jacoby, 1991; Jacoby et al.,
1993; Jennings \& Jacoby, 1993; Toth, Reingold, \& Jacoby, 1994). Although there is some doubt regarding whether familiarity and recollection are truly independent processes (Curran \& Hintzman, 1995; Joordens \& Merikle, 1993), these findings provide relatively strong support for the claim that both intentional and nonintentional processes influence performance on memory tasks.

In summary, there are strong grounds for doubting that the exhaustiveness and exclusiveness criteria can ever be met. If either criterion is violated, one cannot draw valid inferences regarding the conscious status of task knowledge from task dissociations. These problems still hold, even if one satisfies the standard criticisms of the dissociation paradigm (e.g., Holender, 1986; Perruchet \& Pacteau, 1990) and uses more sensitive measures of awareness, such as recognition. Neither dissociations nor associations can provide evidence for implicit or explicit learning. This conclusion is of major concern, given that virtually all of the evidence for implicit learning has relied on this logic. ${ }^{2}$

\section{Alternative Approaches for Inferring the "Implicit" Status of Task Knowledge}

Given the problems with the task dissociation paradigm, future research should focus on attempting to develop alternative strategies for evaluating whether episodic and abstract knowledge can exert an implicit influence on task performance. Several strategies are currently available, including secondary task methodology (Curran \& Keele, 1993), measures of perceptual fluency (Buchner, 1994), analysis of false recognition data (Whittlesea \& Dorken, 1993a), and the process dissociation procedure (Neal et al., 1995). Unlike the task dissociation procedure, these strategies do not attempt to operationalize the construct of awareness. Secondary tasks are used to operationalize attentional allocation, whereas measures of perceptual fluency, false recognition data, and the process dissociation procedure are used to operationalize intentional control. Although all of these these approaches have limitations, they do use constructs (attention or intention) that are more easily operationalized than awareness, and they may offer greater explanatory power.

Secondary tasks are used to block the allocation of attention to task performance. Cohen, Ivry, and Keele (1990) demonstrated that subjects could learn complex repeating sequences under dual-task conditions and that this learning was dissociated from performance on a measure of declarative knowledge. Furthermore, Curran and Keele (1993) demonstrated that performance was associated with declarative knowledge under single-task conditions and was dissociated from declarative knowledge under dual-task conditions. Curran and Keele's interpretation is that both attentional and nonattentional forms of learning occur under single-task conditions, but that only nonattentional learning occurs under dual-task conditions. However, these findings are not conclusive, because they 
still rely on the logic of association and dissociation, and they are subject to all of the problems with the task dissociation procedure raised earlier.

Buchner (1994) evaluated whether prior knowledge can exert a nonintentional influence on task performance by using reaction time as a measure of familiarity. Familiarity is commonly assumed to reflect the fluency with which an item can be processed (Johnston, Dark, \& Jacoby, 1985). Reaction time can be used to measure familiarity, because highly familiar items should be processed more quickly and fluently than unfamiliar items. In relation to recognition memory, it has been found that reaction times are faster for items identified as "old" than for items identified as "new" (Johnston et al., 1985). The difference in reaction time for "old" and "new" responses is taken to suggest that familiarity mediates recognition judgments. If familiarity also mediates categorization judgments (e.g., Servan-Schreiber \& Anderson, 1990), reaction times should be shorter for "grammatical" responses than for "nongrammatical" responses (Buchner, 1994). Using a perceptual clarification procedure, Buchner found that recognition responses were related to reaction time, as predicted, but that categorization decisions were not. These findings suggest that familiarity may not mediate categorization judgments, and that prior knowledge may not exert an unintentional influence on task performance.

However, Buchner's (1994) conclusion rests on the assumption that familiarity reflects the contribution of perceptual fluency. Within the categorization literature, familiarity is more commonly viewed as reflecting the global similarity between the training and test items (Medin \& Schaffer, 1978; Nosofsky, 1986; see also Jacoby, 1991) rather than perceptual fluency. Perceptual clarification procedures may not provide an adequate test of hypothesized links between familiarity and categorization if familiarity reflects the operation of global matching processes, rather than perceptual fluency.

Whittlesea and Dorken (1993a) used false recognition data to test whether prior knowledge can exert a nonintentional influence on task performance. Whittlesea and Dorken found that subjects were more likely to falsely recognize grammatical than nongrammatical items. These data suggest that grammatical knowledge can exert a nonintentional influence on recognition responses. The false alarms had to be nonintentional, because the subjects would have called the items new if they could recollect the training items. Furthermore, the effect of false recognition interacted with the processing context. False recognition was stronger when the test context matched the training context. The sensitivity of false recognition to encoding specificity effects suggests that these nonintentional influences of prior experience reflected the use of episodic knowledge.

Jacoby's (1991) process dissociation procedure has also been used to test whether prior knowledge can exert a nonintentional influence on task performance. Although Jacoby and his colleagues (e.g., Jacoby et al., 1993) often refer to intentional uses of memory as "conscious," and nonintentional uses of memory as "unconscious," the process dissociation procedure deals with the issue of intention, and not awareness. As stated previously, process dissociation involves comparing performance under conditions in which intentional processes act in concert with each other with a condition in which they act in opposition. The experimenter can draw inferences regarding the contribution of both types of memory directly from the experimental manipulations, or by using the process estimation procedure.

Unfortunately, a number of authors have pointed to problems in the process estimation procedure. Problems already identified include potential violations of the independence assumption (Curran \& Hintzman, 1995; Joordens \& Merikle, 1993), changes in the conditional probability of relying on intentional and nonintentional memory across inclusion and exclusion tasks (Graf \& Komatsu, 1994), and nonlinearities in the solution space for the estimates of nonintentional memory (Neal, 1996). However, these problems are confined to the estimation procedure, and do not compromise the validity of inferences drawn directly from the effects of the inclusion and exclusion tasks. For these reasons, it may be advisable for researchers to draw inferences regarding the contribution of both types of memory from their raw data, rather than rely on the process estimation procedure.

Neal et al. (1995) adapted the process dissociation procedure for use with categorization tasks by developing exclusion tasks that manipulated intentional uses of memory independently of intentional uses of rule knowledge. These memory-exclusion and rule-exclusion tasks allowed Neal et al. to draw inferences regarding the contribution of intentional uses of rule knowledge, intentional uses of memory, and nonintentional uses of memory to categorization performance. In a financial decisionmaking task, subjects had to identify whether a loan was a sound, average, or poor investment, and to decide whether to approve the loan or not. During the training phase, the subjects learned that sound and average investments had their loans approved, whereas poor investments had their loans rejected. In the test phase, the exclusion groups were told to reject applications from average investments. The memory-exclusion group was told to use recollection to identify the average investments and reject their loans, whereas the rule-exclusion group was told to use the rule to identify the average investments and reject their loans. Neal et al. found that subjects mistakenly approved loans to items that were similar to previously seen average investments under both memory-exclusion and rule-exclusion conditions. Even giving the subjects practice at using the rule could not eliminate the influence of similarity. The inability of subjects to control the intrusion of prior items suggests that memory for prior instances may exert a nonintentional influence on categorization performance. These nonintentional influences of memory were found to remain invariant across encoding manipulations that had the opposite effects on intentional uses of memory and intentional uses of rule knowledge. Similar findings were reported by Allen and Brooks 
(1991), who found that subjects were unable to control the intrusion of prior items when instructed to do so.

Collectively, the findings discussed in the present section provide some preliminary evidence to support the claim that episodic knowledge can exert a nonintentional influence on task performance. This line of research appears more promising for investigating the implicit status of categorization performance than do existing research strategies, which are based on the logic of task association and dissociation. One of the reasons for the current progress may be that these more recent techniques do not address the issue of awareness. The following section reviews the role of consciousness in psychological theory and concludes that the use of consciousness as an explanatory construct is questionable. It is argued that future research should focus on issues such as intention, rather than awareness.

\section{The Role of Consciousness in Psychological Theory}

The ongoing debate over implicit learning can be seen as part of a broader debate over the status of consciousness as an explanatory construct within cognitive psychology. The debate over the role of consciousness is somewhat paradoxical. On the one hand, virtually all information processing models appear to assume that complex forms of information processing occur outside of awareness, yet on the other hand, many cognitive psychologists have been extremely sceptical of claims for phenomena such as implicit learning.

Most theorists appear to accept the distinction between conscious and unconscious information processing. For example, few cognitive psychologists would claim that people have introspective access to the algorithms used in depth perception, social judgment, or speech production. The computational descriptions of these phenomena are so complex, and sufficiently at odds with our phenomenal experience, that it appears that these computational processes must be carried out unconsciously. Indeed Lewicki and Hill (1989) claim that

the ability of the human cognitive system to nonconsciously acquire information is a general metatheoretical assumption of almost all contemporary cognitive psychology. Moreover, this assumption is so necessary that it is enthymematically present in almost every piece of research on human information processing published over the past two decades and is indirectly reflected in most of the experimental paradigms developed by cognitive psychologists. (p. 239)

The scepticism regarding the use of consciousness as an explanatory construct stems from the functional status of cognitive psychology. The goal of cognitive psychology is to provide a computational analysis of mental phenomena. Consciousness is thought to have no role in this analysis, because it is commonly assumed that the phenomenal properties of conscious experience (e.g., the smell of a rose or the taste of wine) cannot be expressed in computational terms and cannot add to the functional description of mental phenomena. One of the major reasons why implicit learning is so controversial may be that it appears to demonstrate that consciousness does play a functional role in human behavior. If implicit learning exists, this suggests that there are qualitative differences between information processing "inside" and "outside" of consciousness. This position is controversial, because it is unclear how consciousness could be expressed within a computational theory. Indeed, several authors have argued that these difficulties demonstrate that the traditional functionalist approach to cognitive psychology is flawed. Marcel (1988), for example, argues that the existence of unconscious cognition, in the form of implicit learning or subliminal perception, demonstrates that mental phenomena cannot be explained solely by reference to a computational level of analysis (see also Searle, 1990). Such conclusions are deeply disturbing, because they run counter to the fundamental assumptions of cognitive psychology.

In the present paper, however, we have argued that the empirical case for the distinction between conscious and unconscious forms of learning is much weaker than previously thought. There is no technique currently available that allows the experimenter to draw valid conclusions regarding the existence, or otherwise, of qualitative differences between conscious and unconscious forms of learning. Instead, we suggest that dissociations between task performance and declarative knowledge reflect differences in the retrieval cues and processing operations used for the two tasks, rather than the operation of conscious and unconscious forms of information processing. The absence of any valid technique for operationalizing the distinction between conscious and unconscious learning suggests that consciousness may not be a useful construct within implicit learning paradigms. In many respects, the position that we have adopted here is similar to that developed by Dennett (1993). Dennett argued on philosophical grounds that the distinction between conscious and unconscious cognition is incoherent, and that phenomena such as subliminal perception can be explained without making reference to qualitative distinctions between conscious and unconscious processes (see Allport, 1988, for a similar argument).

Collectively, these arguments suggest that the debate over implicit learning should be repositioned, and that future research should examine the role of intention, rather than awareness. Dual-process theories of memory provide both a strong theoretical rationale, and a set of experimental techniques (process dissociation and false recognition) for investigating the role of intention. In particular, research conducted within the context of dual-process theories of memory can proceed by examining the sensivity of estimates of intentional and nonintentional uses of memory to experimental manipulations thought to have differential effects on the two processes (e.g., see Jacoby et al., 1993). However, it must be acknowledged that the evidence supporting the distinction between intentional and nonintentional forms of categorization is still only preliminary. In particular, it may be possible to explain 
the results of process dissociation studies without introducing a distinction between intentional and nonintentional forms of cognition. Exclusion false alarms could be produced by the intentional retrieval of degraded memories, or by source amnesia, rather than by nonintentional uses of memory. ${ }^{3}$ For this reason, it may be necessary to obtain converging evidence regarding the role of intention from other paradigms. One possibility may be to compare the effects of test phase instructions that require subjects to make use of prior instances or rules (direct instructions) with those that do not require subjects to make use of instances or rules (indirect instructions), under conditions in which all other cues are equated across the two conditions (the "retrieval intentionality criterion": Schacter, Bowers, \& Booker, 1989). If experimental variables (e.g., secondary tasks) can be found which have different effects on performance under direct and indirect test conditions, this would suggest that intention constitutes a useful explanatory construct within implicit learning paradigms.

\section{Conclusion}

After 20 years of controversy, it seems clear that subjects can perform implicit learning tasks either by using memory for prior instances or by reference to general knowledge of the structural characteristics of the task. However, researchers still disagree over the representation of these types of knowledge in memory, and over whether either or both can exert an implicit influence on task performance. Much of the difficulty of resolving the implicit status of task knowledge can be traced back to the limited range of research strategies that have been used in the literature. The use of a wider range of research strategies, which shift the focus away from awareness and onto intention, may help advance our understanding of the phenomenon.

\section{REFERENCES}

Allen, S. W., \& Brooks, L. R. (1991). Specializing the operation of an explicit rule. Journal of Experimental Psychology: General, 120, 3-19.

AllporT, A. (1988). What concept of consciousness? In A. J. Marcel \& E. Bisiach (Eds.), Consciousness in contemporary science (pp. 159-182). New York: Oxford University Press.

BERRY, D. C. (1994). Implicit learning: Twenty-five years on. In C. Umiltà \& M. Moscovitch (Eds.), Attention and performance $X V$. Conscious and nonconscious information processing (pp. 755-782). Cambridge, MA: MIT Press.

Berry, D. C., \& Broadbent, D. E. (1984). On the relationship between task performance and verbalizable knowledge. Quarterly Journal of Experimental Psychology, 36A, 209-231.

Brody, N. (1989). Unconscious learning of rules: Comment on Reber's analysis of implicit learning. Journal of Experimental Psychology: General, 118, 236-238.

Brooks, L. R. (1978). Nonanalytic concept formation and memory for instances. In E. Rosch \& B. Lloyd (Eds.), Cognition and categorization (pp. 169-211). Hillsdale, $\mathrm{NJ}$ : Erlbaum.

Brooks, L. R., \& VoKey, J. R. (1991). Abstract analogies and abstracted grammars: Comments on Reber (1989) and Mathews et al (1989). Journal of Experimental Psychology: General, 120, 316323.

BUCHNER, A. (1994). Indirect effects of synthetic grammar learning in an identification task. Journal of Experimental Psychology: Learning, Memory, \& Cognition, 20, 550-566.

Cohen, A., IVry, R., \& KeEle, S. W. (1990). Attention and structure in sequence learning. Journal of Experimental Psychology: Learning, Memory, \& Cognition, 16, 17-30.

Curran, T., \& Hintzman, D. L. (1995). Violations of the independence assumption in process dissociation. Journal of Experimental Psychology: Learning, Memory, \& Cognition, 21, 531-547.

Curran, T., \& Keele, S. W. (1993). Attentional and nonattentional forms of sequence learning. Journal of Experimental Psychology: Learning, Memory, \& Cognition, 19, 189-202.

Debner, J. A., \& JACOBY, L. L. (1994). Unconscious perception: Attention, awareness, and control. Journal of Experimental Psychology: Learning, Memory, \& Cognition, 20, 304-317.

DenNetT, D. C. (1993). Consciousness explained. Harmondsworth, U.K.: Penguin.

Dienes, Z., \& Berry, D. C. (1997). Implicit learning: Below the subjective threshold. Psychonomic Bulletin \& Review, 4, 3-23.

Dienes, Z., Broadbent, D. E., \& Berry, D. C. (1991). Implicit and explicit knowledge bases in artificial grammar learning. Journal of Experimental Psychology: Learning, Memory, \& Cognition, 17, 875-887.

Dulany, D. E., Carlson, R. A., \& Dewey, G. I. (1984). A case of syntactical learning and judgment: How conscious and how abstract? Journal of Experimental Psychology: General, 113, 541-555.

ERDELY1, M. H. (1986). Experimental indeterminacies in the dissociation paradigm of subliminal perception. Behavioral \& Brain Sciences, 9, 30-31.

Graf, P., \& Komatsu, S. (1994). Process dissociation procedure: Handle with caution! European Journal of Cognitive Psychology, 6, 113-129.

Higham, P., \& Vokey, J. R. (1994). Recourse to stored exemplars is not necessarily explicit: A comment on Knowlton, Ramus, \& Squire (1992). Psychological Science, 5, 59-60.

HinTZMAN, D. L. (1986). "Schema abstraction" in a multiple trace memory model. Psychological Review, 93, $411-428$.

HolENDER, D. (1986). Semantic activation without conscious identification in dichotic listening, parafoveal vision, and visual masking: A survey and appraisal. Behavioral \& Brain Sciences, 9, 1-66.

Humphreys, M. S., Wiles, J., \& DenNis, S. (1994). Toward a theory of human memory: Data structures and access processes. Behavioral \& Brain Sciences, 17, 655-692.

JACOBY, L. L. (1983). Remembering the data: Analyzing interactive processes in reading. Journal of Verbal Learning \& Verbal Behavior, 22, 485-508.

JACOBY, L. L. (1991). A process dissociation framework: Separating automatic from intentional uses of memory. Journal of Memory $\&$ Language, 30, 513-541.

JACOBY, L. L., BAKER, J. G., \& BROOKS, L. R. (1989). Episodic effects on picture identification: Implications for theories of concept learning and theories of memory. Journal of Experimental Psychology: Learning, Memory, \& Cognition, 15, 275-281.

JACOBY, L. L., \& BROOKS, L. R. (1984). Nonanalytic cognition: Memory, perception, and concept learning. In G. H. Bower (Ed.), The psychology of learning and motivation (Vol, 18, pp. 1-47). New York. Academic Press.

JACOBY, L. L., \& DALlAs, M. (1981). On the relationship between autobiographical memory and perceptual learning. Journal of Experimental Psychology: General, 110, 306-340.

Jacoby, L. L., Toth, J. P., \& Yonelinas, A. P. (1993). Separating conscious and unconscious influences of memory: Measuring recollection. Journal of Experimental Psychology: General, 122, 139-154.

JenNIngs, J. M., \& JACOBY, L. L. (1993). Automatic versus intentional uses of memory: Aging, attention and control. Psychology \& Aging, 8, 283-293

Johnston, W. A., Dark, V. J., \& Jacoby, L. L. (1985). Perceptual fluency and recognition judgments. Journal of Experimental Psychology: Learning, Memory, \& Cognition, 11, 3-11.

Joordens, S., \& MERIKLE, P. M. (1993). Independence or redundancy? Two models of conscious and unconscious influences. Journal of Experimental Psychology: General, 122, 462-467.

Knowlton, B. J., Ramus, S. J., \& Squire, L. R. (1992). Intact artificial grammar learning in amnesia: Dissociation of classification 
learning and explicit memory for specific instances. Psychological Science, 3, 172-179.

LEWICKI, P., \& HILL, T. (1989). On the status of nonconscious processes in human cognition: Comment on Reber. Journal of Experimental Psychology: General, 118, 239-241.

Lewicki, P., HILL, T., \& Bizot, E. (1988). Acquisition of procedural knowledge about a pattern of stimuli that cannot be articulated. Cognitive Psychology, 20, 24-37.

Mandier, G. (1980). Recognizing: The judgement of previous occurrence. Psychological Review, 87, 252-271.

MARCEL, A. J. (1988). Phenomenal experience and functionalism. In A. J. Marcel \& E. Bisiach (Eds.), Consciousness in contemporary science (pp. 121-158). New York: Oxford University Press

MATHEWs, R. C. (1991). The forgetting algorithm: How fragmentary knowledge of exemplars can abstract knowledge. Journal of Experimental Psychology: General, 120, 117-119.

Mathews, R. C., \& Roussel, L. G. (1993). Automatic abstraction of stimulus structure from episodes: Comment on Whittlesea and Dorken (1993). Journal of Experimental Psychology: General, 122, $397-400$.

McAndrews, M. P., \& Moscovitch, M. (1985). Rule-based and exemplar-based classification in artificial grammar learning. Memory \& Cognition, 13, 469-475.

MEDIN, D. L. (1986). Comment on "Memory storage and retrieval processes in category learning." Journal of Experimental Psychology: General, 115, 373-381.

Medin, D. L., Dewey, G. I., \& MurPhy, T. D. (1983). Relationships between item and category learning: Evidence that abstraction is not automatic. Journal of Experimental Psychology: Learning, Memory, \& Cognition, 9, 607-625

MEdin, D. L., \& SChafFer, M. M. (1978). Context theory of classification learning. Psychological Review, 85, 207-238.

Medin, D. L., \& Schwanenflugel, P. J. (1981). Linear separability in classification learning. Journal of Experimental Psychology: Human Learning \& Memory, 7, 355-368.

Merikle, P. M., \& Cheesman, J. (1986). Consciousness is a "subjective" state. Behavioral \& Brain Sciences, 9, 42-43.

Merikle, P. M., \& Reingold, E. M. (1991). Comparing direct (explicit) and indirect (implicit) measures to study unconscious memory. Journal of Experimental Psychology: Learning, Memory, \& Cognition, 17, 224-233.

Morris, C. D., Bransford, J. D., \& Franks, J. J. (1977). Levels of processing versus transfer appropriate processing. Journal of Verbal Learning \& Verbal Behavior, 16, 519-533.

MORTON, J. (1969). Interaction of information in word recognition. Psychological Review, 76, 165-178.

NEAL, A. (1996). Intentional and nonintentional uses of enisodic knowledge in categorisation: An alternative perspective on implicit learning. Unpublished doctoral dissertation, University of New South Wales, Sydney.

Neal, A., Hesketh, B., \& Andrews, S. (1995). Instance-based categorization: Automatic versus intentional forms of retrieval. $\mathrm{Mem}$ ory \& Cognition, 23, 227-242.

NosofSKy, R. M. (1986). Attention, similarity and the identificationclassification relationship. Journal of Experimental Psychology: General, 115, 39-57.

Nosofsky, R. M. (1988). Exemplar-based accounts of relationships between classification, recognition and typicality. Journal of Experimental Psychology: Learning, Memory, \& Cognition, 14, 700708

Perruchet, P. (1994a). Defining the knowledge units of a synthetic language: Comment on Vokey and Brooks (1992). Journal of Experimental Psychology: Learning, Memory, \& Cognition, 20, 223228.

Perruchet, P. (1994b). Learning from complex rule-governed environments: On the proper functions of nonconscious and conscious processes. In C. Umiltà \& M. Moscovitch (Eds.), Attention and performance $X V$ : Conscious and nonconscious information processing (pp. 811-835). Cambridge MA: MIT Press.

Perruchet, P., \& Amorim, M. (1992). Conscious knowledge and changes in performance in sequence learning: Evidence against dis- sociation. Journal of Experimental Psychology: Learning, Memory, \& Cognition, 18, 785-800.

Perruchet, P., Gallego, J., \& Pacteau, C. (1992). A reinterpretation of some earlier evidence for the abstractness of implicitly acquired knowledge. Quarterly Journal of Experimental Psychology, 44A, 193-210.

Perruchet, P., \& Pacteau, C. (1990). Synthetic grammar learning: Implicit rule abstraction or explicit fragmentary knowledge? Journal of Experimental Psychology: General, 119, 264-275.

Posner, M. I., \& KeEle, S. W. (1968). On the genesis of abstract ideas. Journal of Experimental Psychology, 77, 353-363.

Ratcliff, R., VAN ZANDT, T., \& McKoon, G. (1995). Process dissociation, single process theories, and recognition memory. Journal of Experimental Psychology: General, 124, 352-374.

REBER, A. S. (1989). Implicit learning and tacit knowledge. Journal of Experimental Psychology: General, 118, 219-235.

REBER, A. S., \& ALLEN, R. (1978). Analogic and abstraction strategies in synthetic grammar learning: A functionalist interpretation. Cognition, 6, 189-221.

Reber, A. S., Allen, R., \& Regen, S. (1985). Syntactical learning and judgment, still unconscious and still abstract: Comment on Dulany, Carlson, and Dewey. Journal of Experimental Psychology: General, $114,17-24$

REBER, A. S., \& LEwIS, S. M. (1977). Toward a theory of implicit learning: The analysis of the form and structure of a body of tacit knowledge. Cognition, 5, 333-361.

ReINGOLD, E. M., \& MeRIKLE, P. M. (1988). Using direct and indirect measures to study perception without awareness. Perception \& Psychophysics, 44, 563-575.

RothKoPf, E. Z., \& DASHEN, M. L. (1995). Particularization: Inductive speeding of rule-governed decisions by narrow application experience. Journal of Experimental Psychology: Learning, Memory, \& Cognition, 21, 469-482.

SCHACTER, D. L., Bowers, J., \& BOOKER, J. (1989). Intention, awareness, and implicit memory: The retrieval intentionality criterion. In S. Lewandowsky, J. C. Dunn, \& K. Kirsner (Eds.), Implicit memory: Theoretical issues (pp. 47-65). Hillsdale, NJ: Erlbaum.

SeAger, C. A. (1994). Implicit learning. Psychological Bulletin, 115, $163-196$.

SEARLE, J. R. (1990). Minds, brains and programs. In M. A. Boden (Ed.), The philosophy of artificial intelligence (pp. 67-88). Oxford: Oxford University Press.

Servan-Schreiber, E., \& ANderson, J. R. (1990). Learning artificial grammars with competitive chunking. Journal of Experimental Psychology: Learning, Memory, \& Cognition, 16, 592-608.

Shanks, D. R., \& ST. JoHN, M. F. (1994). Characteristics of dissociable human learning systems. Behavioral \& Brain Sciences, 17, 367-447.

Tотн, J. P., REINGold, E. M., \& JACOBY, L. L. (1994). Toward a redefinition of implicit memory: Process dissociations following elaborative processing and self-generation. Journal of Experimental Psychology: Learning, Memory, \& Cognition, 20, 290-303.

Tulving, E., \& ThOMson, D. M. (1973). Encoding specificity and retrieval processes in episodic memory. Psychological Review, 80, 352-373.

VOKEY, J. R., \& Brooks, L. R. (1992). The salience of item knowledge in learning artificial grammars. Journal of Experimental Psychology: Learning, Memory, \& Cognition, 18, 328-344.

VOKEY, J. R., \& BROOKs, L. R. (1994). Fragmentary knowledge and the processing-specific control of structural sensitivity. Journal of Experimental Psychology: Learning, Memory, \& Cognition, 20, 1504 1510.

Wattenmaker, W. D. (1991). Learning modes, feature correlations and memory-based categorization. Journal of Experimental Psychology: Learning, Memory, \& Cognition, 17, 908-923.

WATTENMAKER, W. D. (1993). Incidental concept learning, feature frequency, and correlated properties. Journal of Experimental Psychology: Learning, Memory, \& Cognition, 19, 203-222.

Whittlesea, B. W. A. (1987). Preservation of particular experiences in the representation of general knowledge. Journal of Experimental Psychology: Learning, Memory, \& Cognition, 13, 3-17.

Whittlesea, B. W. A., \& Dorken, M. D. (1993a). Incidentally, things 
in general are particularly determined: An episodic-processing account of implicit learning. Journal of Experimental Psychology: General, 122, 227-248.

WhitTlesea, B. W. A., \& Dorken, M. D. (1993b). Potential power of coding particular experiences: Reply to Mathews and Roussel (1993). Journal of Experimental Psychology: General, 122, 401-404.

\section{NOTES}

1. The ability of episodic models to perform abstraction does raise the problem of distinguishing between abstractive and episodic accounts, since the models are essentially computationally equivalent. For this reason, future research may need to develop a clearer conceptualization of what constitutes an abstractive, as opposed to episodic, explanation of learning. One possibility is to examine the role of contextual binding during retrieval (Humphreys, Wiles, \& Dennis, 1994). According to this view, a task could be classified as episodic if retrieval involved the use of a three-way binding between the retrieval cue, prior items, and context. On the other hand, a task could be classified as abstractive if retrieval did not involve the use of context and simply required a two-way binding between the retrieval cue and the prior items. We thank Michael Humphreys for this suggestion.

2. It could be argued that the fact that task dissociations do not prove the existence of separate knowledge systems does not eliminate them as an interesting phenomenon (we thank Robert Mathews for this suggestion). However, we believe that the inability of task dissociations to prove the existence of separate knowledge systems does make these dissociations largely uninteresting. The episodic account suggests that measures of performance and declarative knowledge will be dissociated, simply because they involve different retrieval cues.

3. We thank Pierre Perruchet for this suggestion (see also Ratcliff, Van Zandt, \& McKoon, 1995)

(Manuscript received January 1, 1996; revision accepted for publication May 17, 1996.) 